\title{
APPLICATION OF BRDU IN STUDYING POSTNATAL NEUROGENESIS
}

\author{
Vanya Goranova \\ Department of Anatomy, Histology and Embryology, Medical University of Varna
}

\begin{abstract}
PURPOSES: We applied the thymidine analog, 5-bromo-2'-deoxyuridine (BrdU), that labels DNA to study neurogenic events in many brain regions of the postnatal mammalian brain and to evaluate its paradigms, pitfalls and limitations.

MATERIAL AND METHODS: Different BrdU paradigms and various time points between postnatal day P0 and P51 were applied to Wistar rats. Paraffin sections for single or double BrdU immunohistochemistry combined with neuronal markers nestin, $\mathrm{Dcx}, \mathrm{MAP}(2)$ and NeuN by peroxidase or fluorescence labeling were examined. Sections were analyzed using standard or confocal microscopy and stereology.

RESULTS: Peak neurogenesis was found out in the dentate gyrus during the first postnatal week of life with a progressive decline after $P 9$. Areas with substantial neurogenesis such as subventricular zone of the lateral ventricles, subgranular zone of the hippocampal dentate gyrus, and cerebellum were observed. By P14 to P18, cell proliferation was reduced in most brain areas with exception of the dentate gyrus. Numerical densities of $\mathrm{BrdU}(+)$ cells in all evaluated brain regions were higher in the younger animals and decreased with age. Most newly-enerated cells became nonneuronal cells (endothelial and glial cells). BrdU colocalization with neuronal markers was rarely found out mainly within the dentate gyrus and cerebellum.

CONCLUSION: In the developing rat brain, BrdU labeling showed regional distribution. It was dose-, ageand survival time-dependent. Our findings provided useful information on BrdU application in the study of postnatal neurogenesis.
\end{abstract}

Key words: BrdU, developing rat brain, confocal microscopy, neuronal markers, neurogenesis

\section{INTRODUCTION}

In the mammalian central nervous system (CNS), neurogenesis occurs mainly prenatally but continues in restricted regions during the postnatal life. It is intensive during the first two weeks after

\footnotetext{
Address for correspondence:

Vanya Goranova, $M D, P h D$

Medical University of Varna,

Dept. of Anatomy, Histology and Embryology,

Medical University of Varna,

55 Marin Drinov Str., 9002 Varna, Bulgaria

E-mail:vanya.goranova@mu-varna.bg

Phone: +35952677079
}

Received: August 30, 2012

Accepted: January 15, 2013 birth picking at P7-P9. Thereafter, neurogenesis is significantly diminished and remains longlife in the subventricular zone (SVZ) of the lateral ventricle and the subgranular zone (SGZ) of the dentate gyrus (DG) $(3,13)$. Actually, adult neurogenesis repeats in a similar way the stages of generation and functional integration of new neurons like the developing brain. Therefore, data on early postnatal neurogenensis may be helpful to elucidate basic neurogenic mechanisms in the adult brain.

The use of the thymidine analog BrdU has become a valuable tool for studying neurogenesis in the adult mammalian brain, including in human $(6,8,11)$. BrdU immunohistochemistry is currently the most used technique for birth dating and cell proliferation monitoring that is one of the most 
important processes involved in CNS development $(1,10,15)$. However, the use of BrdU for studying neurogenesis is not without pitfalls and limitations. As a thymidine analog, BrdU is a marker of DNA synthesis and not a marker of the S-phase of the cell cycle. Moreover, BrdU is a toxic substance that alters DNA stability, lengthens the cell cycle and triggers cell death. It also has mitogenic, transcriptional and translational effects with profound consequences on neurogenesis $(7,12,13)$. Therefore, the application of $\mathrm{BrdU}$ requires distinguishing the cell proliferation from other processes involving DNA synthesiss such as DNA repair, gene duplication and abortive cell cycle reentry.

The goal of this investigation was to provide additional information on BrdU application in the study of early postnatal neurogenic events that may give better insights into adult neurogenesis.

\section{MATERIAL AND METHODS}

All animal experiments were performed in accordance to the institutional guidelines. Wistar rats (BgVV, Berlin) were injected with BrdU (Sigma, St. Louis, MO) using different paradigms (see Fig.
1). Animals were anesthetized and transcardially perfused with 0,01M PBS, followed by $4 \%$ paraformaldehyde in $0,1 \mathrm{M}$ PB. All brains were removed and postfixed in the same fixative. Paraffin sections were processed for standard single or double immunolabeling for peroxidase or fluorescence staining. First antibodies against BrdU, Nestin, Doublecortin (Dcx), MAP(2) and NeuN followed by corresponding biotinylated second antibodies, $\mathrm{ABC}$ reagent and $\mathrm{DAB} / \mathrm{VNR}$ substrate were applied. By fluorescence labeling, Alexa Fluor 594 or 488 and Vectashield mounting medium with DAPI were used. Microscopic analysis of the stained sections was done and digital images were taken. Morphometric counts evaluating BrdU(+) nuclei by peroxidase labeling on P9, P12, P15 and P23 or Dcx(+) cells, BrdU(+) nuclei and $\mathrm{Dcx}(+) / \mathrm{BrdU}(+)$ colocalization on P15 using confocal images were done in a blinded fashion. Values were presented as mean \pm S.E.M.

\section{RESULTS}

We have examined intensity and distribution of BrdU immunostaining in rat brains by different paradigms. By same dosage, longer survival times
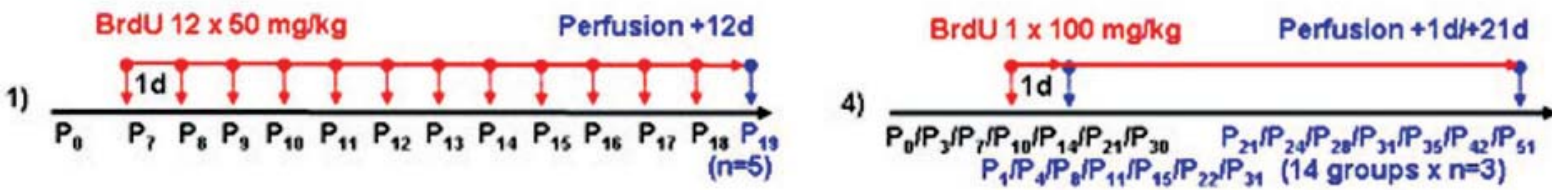

BrdU $1 / 3 \times 100 \mathrm{mg} / \mathrm{kg}$
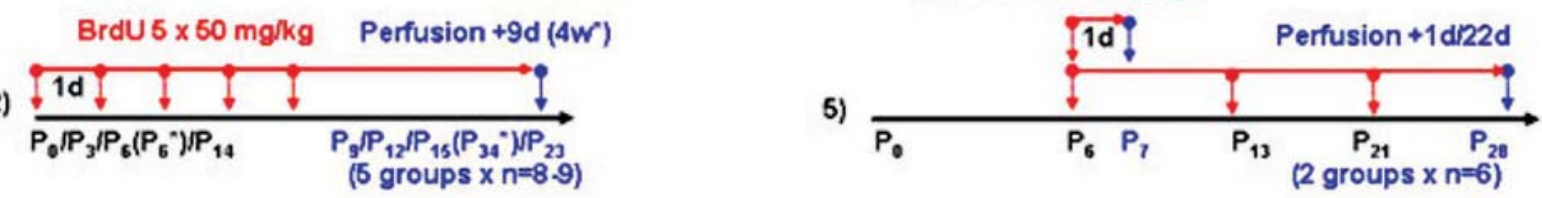

BrdU $3 \times 50 \mathrm{mg} / \mathrm{kg}$ Perfusion $+3 \mathrm{~d}$
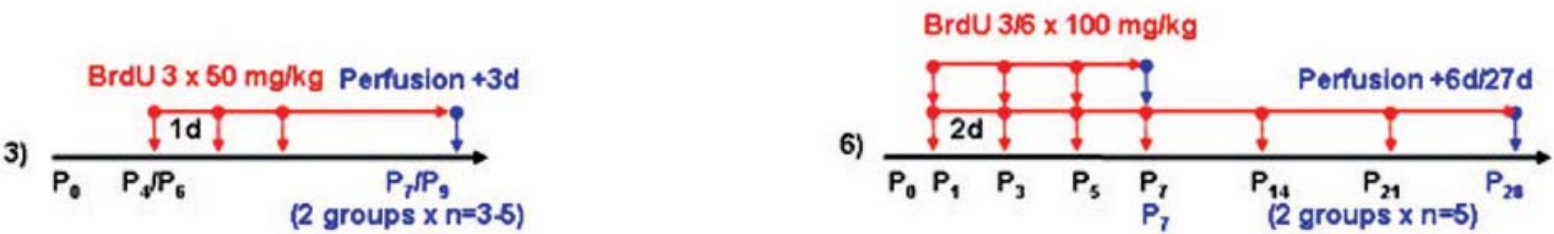

Fig. 1. Six main BrdU paradigms were used in this study. Animals on P0 to P30 were distributed into 26 groups ( $n=3-$ 9 per group) and daily injected with 50 or $100 \mathrm{mg} / \mathrm{kg} \mathrm{BrdU}$ i.p. one or more days. Perfusion was performed at various survival times between 1 to 28 days

Scripta Scientifica Medica, 2013, vol. 45 (1), pp. 24-28

Copyright (C) Medical University - Varna 


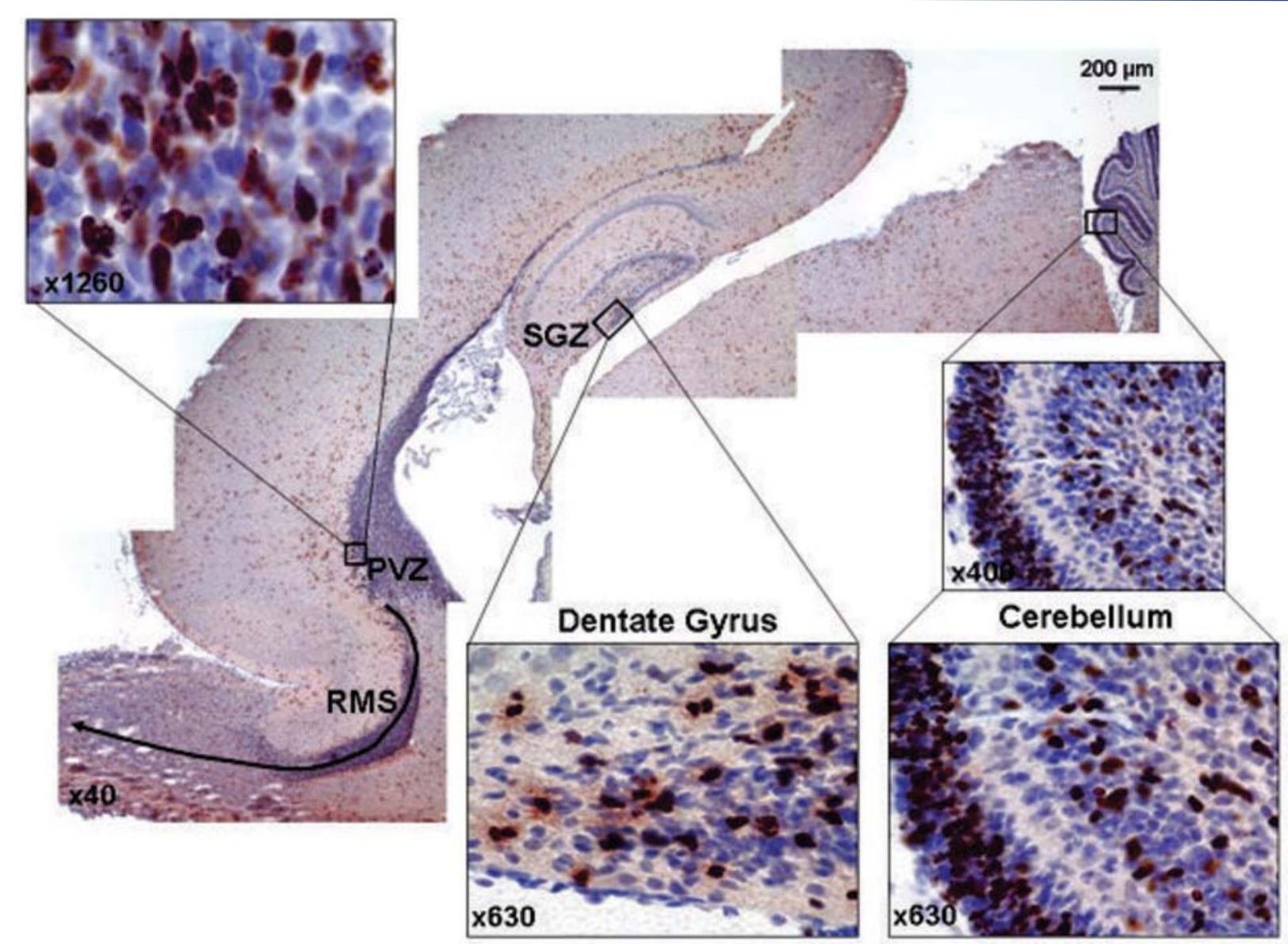

Fig. 2. Micrographs showing a partial sagittal view of a rat brain on P7 after one $100 \mathrm{mg} / \mathrm{kg}$ BrdU injection on P6. An intense immuno(+) staining for BrdU is detected in PVZ of the lateral ventricle, RMS, SGZ of DG and cerebellar cortex. Indirect peroxidase immunohistochemistry for BrdU (VNR substrate) and hematoxylin counterstaining

showed a reduced staining. In our pilot experiment, we have found out that a less intensive BrdU staining was present by lower dosages of BrdU when survival times were equal. Younger animals showed more expressed BrdU positivity in all ten regions examined from various parts of the brain such as frontal and parietal cortices, caudate, thalamus, DG and cerebellum. A very dense staining was detected in many cells of PVZ which were directed by the rostral migratory stream (RMS) into the olfactory bulb, as well as in SGZ of DG and cerebellum (Fig. 2). At the end of the second postnatal week, BrdU expression was restricted mainly to two regions, i.e., the SVZ of the lateral ventricle and SGZ of DG.

Summarized total proliferative scores of $\mathrm{BrdU}(+)$ cell countings in ten brain regions at $\mathrm{P} 9, \mathrm{P} 12$, P15 and P23 demonstrated a significant decrease compared to each previous age group (Fig. 3B), the respective BrdU paradigms were presented in Fig. 3A. We quantified Dcx, BrdU, Dcx/BrdU immuno(+) cells in rat DG on P15 (fig. 3C). We found that $77,87 \%$ of $\mathrm{BrdU}(+)$ nuclei were located in $\mathrm{Dcx}(+)$ cells and more than one half of $\operatorname{Dcx}(+)$ cells, i.e., $51,04 \%$ were positive for BrdU.

Frequent colocalization for $\mathrm{BrdU}(+) / \mathrm{Nestin}(+)$ or $\operatorname{BrdU}(+) / \operatorname{Dcx}(+)$ was found in specific regions such as the SVZ, cerebellum, corpus callosum and DG-SGZ (data not shown). BrdU(+)/MAP(2)(+) and $\mathrm{BrdU}(+) / \mathrm{NeuN}(+)$ colocalizations were seen by later timings. During the first two postnatal weeks, Nestin(+) radial glia and $\operatorname{Dcx}(+)$ cells underwent a considerable reduction in number. $\mathrm{MAP}(2)$ and NeuN immunoexpression increased after P9 and remained constant in older animals. 
A)

BrdU $5 \times 50 \mathrm{mg} / \mathrm{kg}$ Perfusion $+9 \mathrm{~d}$

B)
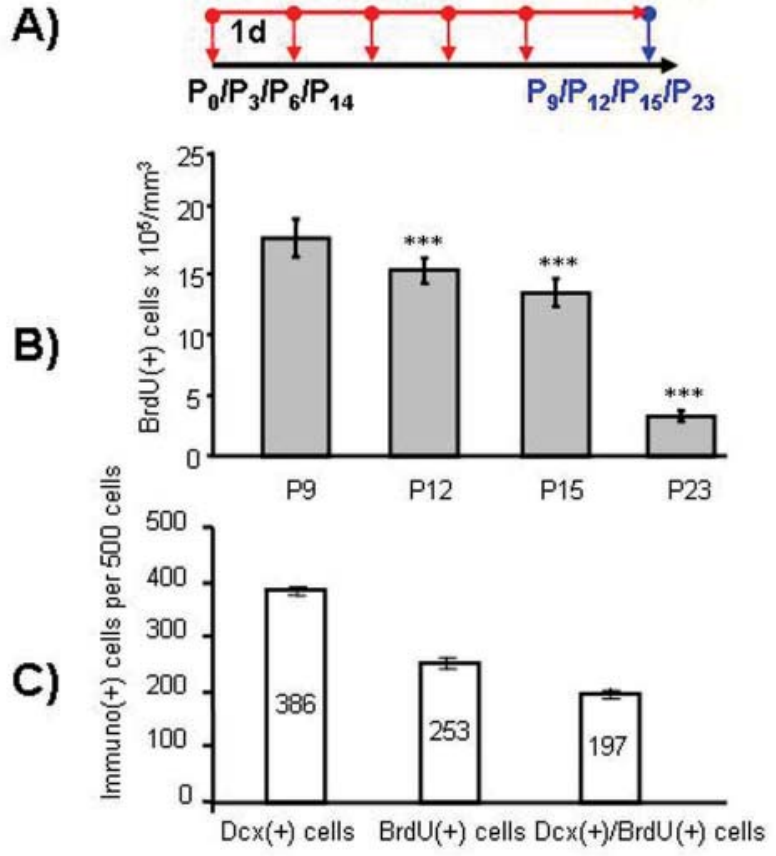

Fig. 3. A) BrdU paradigms for P9, P12, P15 and P23. B) $B r d U(+)$ cell counts in rat brain on P9, P12, P15 and P23 ( $n=5$ for each group). Student's $t$-test, ${ }^{* *} P<0.001$, when compared with each of the previous time point scores. C) $D c x, B r d U, D c x / B r d U$ immuno(+) cell counts in rat $D G$ of $P 15$ group $(n=5)$

\section{DISCUSSION}

As it has been demonstrated in our study of the developing rat brain, cell proliferation as expressed by BrdU immuno(+) nuclei and neuronal differentiation as manifested by neuronal markers such as Dcx, MAP(2) and NeuN display regional distribution and age dependency. BrdU labeling also shows dose and survival time dependency. Reduction in number of Nestin $(+)$ and Dcx cells(+), especially for nestin(+) radial glia in neocortex, together with an increasing expression of $\mathrm{MAP}(2)$ and NeuN are indicative of neuronal migration and differentiation that are still going on during the early postnatal period. Most newly-generated cells in the rat brain become nonneuronal cells (endothelial and glial cells). Our findings on the colocalization of BrdU and Dcx markers in DG on P15 indicate that a higher percentage of the newly-generated cells, i.e., $77,87 \%$ of all $\mathrm{BrdU}(+)$ cells in that particular brain region are positive for Dcx marker and represent young neurons. The remaining lower percentage $(22,13 \%)$ of $\mathrm{BrdU}(+)$ cells negative for Dcx are evidently nonneural cells or, eventually, neural cells in a different phase of their development which do not express any Dcx marker. The $\mathrm{Dcx}(+) / \mathrm{BrdU}(-)$ cells that represent $48,96 \%$ of all $\mathrm{Dcx}(+)$ in DG are obviously generated before P6, the first day of BrdU application for P15 paradigm or they may contain a nondetectable amount of BrdU at the moment of perfusion.

We establish that after the first two weeks, postnatal neurogenesis in the rat is considerably reduced and restricted to two brain regions: the SVZ of the lateral ventricle and SGZ of the DG. In both zones a small pool of multipotent neural precursors remain lifelong and may contribute to repair following CNS disorders $(9,13)$.

BrdU application should be conducted with appropriate controls and combined with other relevant tools and techniques for studying the neurogenesis $(1,2,4)$. It requires careful interpretation of the data and comparison with alternative methods of investigation such as proliferative markers, $14 \mathrm{C}$ labeling, retroviral labeling etc. $(3,5,14)$.

\section{CONCLUSIONS}

Early postnatal neurogenesis in the rat brain continues at a high rate during the first two weeks. Thereafter, it is significantly reduced and remains at a low rate in the SVZ of the lateral ventricle and SGZ of the hippocampal formation. BrdU immunohistochemistry is a valuable and reliable assay for studying the postnatal neurogenesis despite some restrictions and pitfalls.

\section{ACKNOWLEDGEMENTS}

This study was supported by BMBF grants 01GZ0305 and PBZ-MN-001/P05/2002/25-28 in Germany. It was conducted at the Dept. of Pediatric Neurology, Charité-Virchow Clinics, Berlin and the Dept. of Pediatric Neurology, Medical Faculty, Dresden. Thanks are due to prof. C. Ikonomidou for experimental design, A. Böhme and F. Pan-Montojo for microscopic assistance. 


\section{REFERENCES}

1. Abrous, D. N., M. Koehl, M. Le Moal. Adult neurogenesis: from precursors to network and physiology.- Physiol. Rev., 85, 2005, No 2, 523-569.

2. Eisch, A. J., C. D. Mandyam. Adult neurogenesis: can analysis of cell cycle proteins move us "Beyond BrdU”?- Curr. Pharm. Biotechnol., 8, 2007, No 3, 147-165.

3. Gould, E. How widespread is adult neurogenesis in mammals?- Nat. Rev. Neurosci., 8, 2007, No 6, 481-488.

4. Hancock, A., C. Priester, E. Kidder, J. R. Keith. Does 5-bromo-2'-deoxyuridine (BrdU) disrupt cell proliferation and neuronal maturation in the adult rat hippocampus in vivo?- Behav. Brain Res., 199, 2009, No 2, 218-221.

5. Kee, N., S. Sivalingam, R. Boonstra, J. M. Wojtowicz. The utility of Ki-67 and BrdU as proliferative markers of adult neurogenesis.- $J$. Neurosci. Methods, 115, 2002, No 1, 97-105.

6. Kuhn, H. G., C. M. Cooper-Kuhn. Bromodeoxyuridine and the detection of neurogenesis.- Curr. Pharm. Biotechnol., 8, 2007, No 3, 127-131.

7. Kuwagata, M., T. Ogawa, T. Nagata, S. Shioda. The evaluation of early embryonic neurogenesis after exposure to the genotoxic agent 5-bromo-2'deoxyuridine in mice.- Neurotoxicology, 28, 2007, No $4,780-789$.

8. Leuner, B., E. R. Glasper, E. Gould. Thymidine analog methods for studies of adult neurogenesis are not equally sensitive.- J. Comp. Neurol., 517, 2009, No 2, 123-133.
9. Lie, D. C., H. Song, S. A. Colamarino, G. L. Ming, F. H. Gage. Neurogenesis in the adult brain: new strategies for central nervous system diseases.Annu. Rev. Pharmacol. Toxicol., 44, 2004, 399-421.

10. Miller, M. W., R. S. Nowakowski. Use of bromodeoxyuridine-immunohistochemistry to examine the proliferation, migration and time of origin of cells in the central nervous system.- Brain Res., 457, 1988, No 1, 44-52.

11. Soriano, E., J. A. Del Rio. Simultaneous immunocytochemical visualization of bromodeoxyuridine and neural tissue antigens.- $J$. Histochem. Cytochem., 39, 1991, No 3, 255-263.

12. Taupin, P. BrdU immunohistochemistry for studying adult neurogenesis: paradigms, pitfalls, limitations, and validation.- Brain Res. Rev., 53, 2007, No 1, 198-214.

13. Taupin, P. Stem cells and regenerative medicine. Volume I. Adult neurogenesis and neural stem cells. 1st ed. New York, Nova Science Publishers, Inc., 2008.

14. Wojtowicz, J. M., N. Kee. BrdU assay for neurogenesis in rodents.- Nat. Protoc., 1, 2006, No 3, 1399-1405.

15. Zhao, M., A. M. Janson Lang. Bromodeoxyuridine infused into the cerebral ventricle of adult mice labels nigral neurons under physiological conditions - a method to detect newborn nerve cells in regions with a low rate of neurogenesis.- $J$. Neurosci. Methods, 184, 2009, No 2, 327-331. 